\title{
Dopamine agonists suppress visual-cortical reflex myoclonus
}

\author{
JA OBESO, J ARTIEDA, T TUÑÓN, ${ }^{*}$ MR LUQUIN, JM MARTINEZ LAGE \\ From the Movement Disorders Unit, Department of Neurology, Clinica Universitaria, University of Navarra, \\ Pamplona, Spain.
}

SUMMARY Two patients with a diagnosis of olivo-ponto-cerebellar atrophy developed cortical reflex myoclonus to visual (flash) and somaesthetic stimuli. Oral treatment with levodopacarbidopa $(1000 / 100 \mathrm{mg})$ or subcutaneous administration of apomorphine $(1 \mathrm{mg})$ abolished the visually-triggered myoclonus, without modifiying reflex myoclonus to electrical or tactile stimulation. Intravenous administration of lisuride $(0.1 \mathrm{mg})$ produced a marked reduction in both types of reflex myoclonus. These results indicate a selective inhibitory effect of dopamine agonist drugs on visual reflex myoclonus of cortical origin.

In recent years the pharmacological basis of myoclonus has been focused on a disorder of cerebral serotoninergic mechanisms. This followed the discovery by Lhermitte et al, ${ }^{2}$ later confirmed by others, ${ }^{3-6}$ that oral treatment with 5-hydroxytryptophan (5-HTP) plus carbidopa produced a reduction in post-anoxic action myoclonus, and the findings of low CSF levels of 5-hydroxyindolacetic acid in similar patients who responded to treatment with 5-HTP or clonazepam. ${ }^{78}$ However, a deficit of serotonin may not be important in other myoclonic disorders ${ }^{910}$ in which other neurotransmitters may be involved. The anti-myoclonic effect of clonazepam, valproic acid or primidone might be due at least partially, to enchancement of cerebral gamma aminobutyric acid (GABA) activity. " A protective effect of apomorphine against visually induced myoclonus has been demonstrated in the baboon..$^{2}$ Quesney et $a l^{13}$ found that in patients with generalised epilepsy, apomorphine produced transient inhibition of spike and wave EEG activity induced by photic stimulation, suggesting a dopamine influence on certain types of myoclonus.

Cortical reflex myoclonus results from abnormal

\footnotetext{
*Present address: Neuropathology section, Hospital de Navarra, Pamplona.

Address for reprint requests: Dr JA Obeso, Neurologia, Clinica Universitaria, Apartado 192, Pamplona, Spain.
}

Received 23 January 1985 and in revised form 9 May 1985. Accepted 17 May 1985 motor cortex activity triggered by sensory input. ${ }^{14}$ is Electrophysiological characteristics of cortical myoclonus are the presence of enhanced cortical evoked potentials, EEG activity time-locked to the muscle jerks and brief EMG discharges. ${ }^{412}$ Clinical and electrophysiological studies indicate the existence of separate mechanisms underlying different types of cortical myoclonus in man. ${ }^{16}$ Such pathophysiological discrimination may also indicate different pharmacological characteristics. For example, cortical reflex myoclonus to somaesthetic stimulation is highly responsive to serotonin agonists, but is not improved by dopaminergic drugs. ${ }^{17}$ We now report a selective effect of levodopa-carbidopa and apomorphine on cortical reflex myoclonus induced by photic stimulation in two patients with olivo-pontocerebellar-atrophy. These two cases were included in a previous report on the pathophysiology of cortical myoclonus. ${ }^{16}$

\section{Method}

Electrophysiological technique A Medelec MS 6 machine was used for electrophysiological investigations. Somatosensory evoked potentials (SEPs) to digital nerve stimulation were recorded from a scalp electrode placed on the hand area $(7 \mathrm{~cm}$ lateral and $2 \mathrm{~cm}$ posterior to the vertex) with the reference electrode on Fz (10-20 EEG international system). Visual evoked potentials (VEPs) to flash stimulation recorded from the scalp over the occipital cortex also were recorded. For reasons explained previously, ${ }^{18}$ we have designated the major cortical potential peaks by their polarity and sequence (N1, P1, N2, etc). The electromyographic (EMG) reflex responses elicited by electrical or flash stimulation were recorded simultane- 


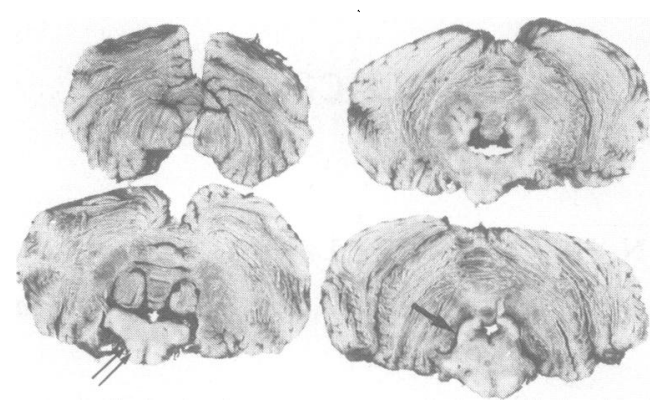

Fig 1 Case 1. Transverse sections of the cerebellum, pons and medulla. Cerebellar white matter is considerably reduced in volume; both dentate nuclei appear normal macroscopically. There is severe atrophy of the pons and middle cerebellar penduncles (single arrow). The brachium conjuctivum is normal bilaterally. In the medulla the normal protusion of the inferior olive has disappeared (double arrow).

ously by surface electrodes placed on biceps brachii or finger flexors. The amplitude of the reflex EMG discharges was measured as the maximum interpeak value on average records before and after each pharmacological test. The duration of the EMG bursts usually remained constant throughout each session.

\section{Case reports}

Case 1 M. R. (Clínica Universitaria No 136647)

A 65-year-old woman with a history of unsteadiness of gait beginning in 1978 was investigated in 1979 for a cerebellar syndrome, but no cause was found. There was no family history of neurological disease. By 1982 the patient had lost facial expression, had difficulty in performing normal daily motor tasks and had frequent falls. She was also incontinent of urine and could not swallow solid food. Examination showed marked rigidity and akinesia as well as dysmetria of the limbs. She could not stand or walk alone. Pursuit eye movements were interrupted and there were ocular dysmetria and "square wave" jerks. Mentation was intact. Cranial nerves, tendon jerks and sensation were all normal. There was no orthostatic hypotension. Brief light touch or pinprick of the skin area innervated by the medial plantar nerve of either foot provoked repetitive jerking of the toes lasting up to 1 second. When the stimulus was applied to the great-toe, or to the second toe or adjacent area, the jerk consisted of extension of the great toe and flexion of the other toes. There was no visible muscle jerking when the patient voluntarily moved the foot or toes. A few months later, when her general condition had deteriorated, it was noticed that touch and particularly pin-prick to the dorsal region of the thumb or forefinger produced brief and repetitive jerks of the forearm and bicep muscles. A CT scan revealed severe brainstem and cerebellar atophy, but the cerebral hemispheres were normal. The clinical diagnosis of sporadic olivo-ponto-cerebellar atrophy was made. In January 1984 EEG showed diffuse theta rhythm at rest, but flash stimulation provoked generalised muscle jerking which blurred the EEG trace, without loss of consciousness. The patient was treated with levodopa-carbidopa (1000/100 mg/day), bromocriptine ( $30 \mathrm{mg} /$ day), lisuride $(5 \mathrm{mg} /$ day) and thyroxine releasing hormone (TRH) iv (10 mg/day) with no improvement in her akinesia and ridigity. She died of pneumonia in March 1984.

On post-mortem examination the brain weighed $1180 \mathrm{~g}$. The brainstem and cerebellum weighed $120 \mathrm{~g}$ (normal control values $160 \pm 10 \mathrm{~g}$ ). Macroscopic examination after fixation showed severe atrophy of the pons, inferior and middle cerebellar peduncles and cerebellum (fig 1). The substantia nigra and locus coeruleus appeared mildly depigmented. The putamen was shrunken bilaterally and the cerebral hemispheres were normal. The spinal cord was normal macroscopically. For histological studies, the whole cerebellum, blocks of several ${ }^{-}$ other brain regions and the spinal cord were embed- $\overrightarrow{\mathbb{D}}$ ded in paraffin wax. Sections were stained with $\frac{\text { Pै }}{\mathrm{D}}$ haematoxylin-eosin, luxol fast-blue, Bielchowsky, Nissl, Holsen and Spilmeyer.

Microscopic examination of the pre-frontal, sensory-motor and visual cortex did not reveal any histological abnormality (fig 2A). Neuronal loss andō fibrillary gliosis were very severe in the putamen? (fig 2B) and moderate in the external globus pallidus. The internal globus pallidus, caudate, thalamus, subthalamus, hypothlamus, red nucleus and geniculate bodies were normal. In the mesencephalon (fig 2C), the substantia nigra (pars reticulata and compacta) and locus coeruleus showed intense gliosis and $20 \%$ decrease of pigmented neurons. Lewy bodies and neurofibrillary tangles were not present. The superior cerebral peduncle was normal. In the pons, neuronal loss and gliosis was widespread and very severe (fig 2-D), sparing only part of the raphe nuclei. The middle and inferior cerebellar peduncles were thin and severely demyelinated. The arcuate fibres also were damaged. The corticospinal tract was slightly pale, but the medial longitudinal fascicle and the central tegmental tract were normal. In the cerebellum (fig 2E) the white matter was severely demyelinated. Purkinje cells were markedly reduced in number and there was Bergmann glia proliferation. The molecular layer showed moderate gliosis; the granular layer was slightly thinner than normal. Within this latter layer, 

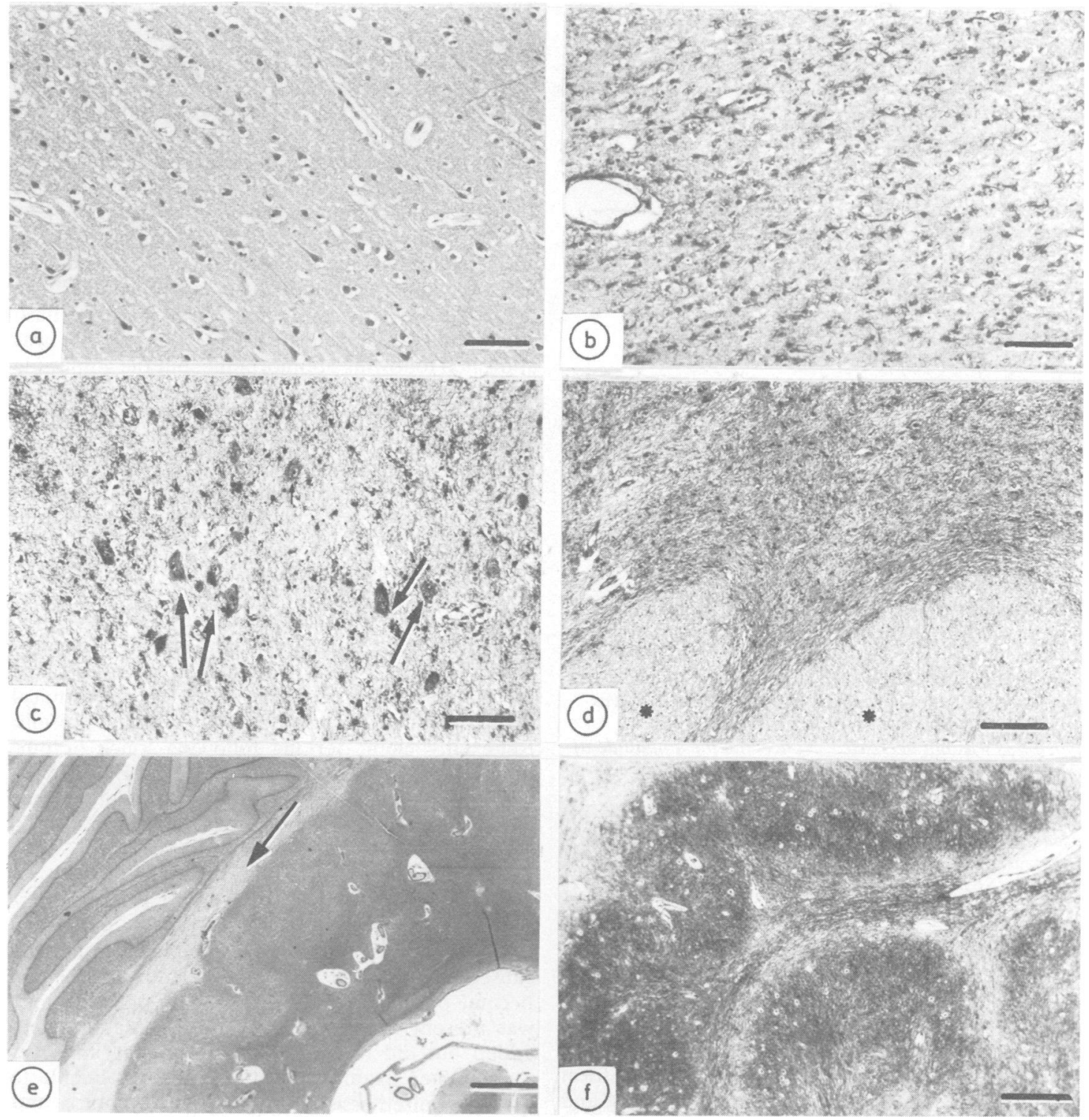

Fig 2 Case 1. Microscopic sections at different levels of the brain. (A) Cortex. Normal somatosensory cortex. One bar = $650 \mu \mathrm{m}$. (B) Putamen. Intense gliosis and marked neuronal loss. Holzer stain, One bar = $650 \mu \mathrm{m}$. (C) Substantia nigra. Severe fibrillary gliosis. Surviving neurons are normal. Holzer stain, One bar $=500 \mu \mathrm{m}$. (D) Pons. Transverse myelinated fibres are considerably reduced (upper half); abundant fibrillary gliosis. Vertical fibres are preserved (asterisk). Holzer stain. One bar $=710 \mu \mathrm{m}$. (E) Cerebellum. Intense demyelination (arrow). The dentate nucleus and its ribbon are normal. Luxol fast blue. One bar = $1600 \mu \mathrm{m}$. (F) Medulla. Striking neuronal loss and fibrillary gliosis of the inferior olive. Holzer stain. One bar $=710 \mu \mathrm{m}$. 


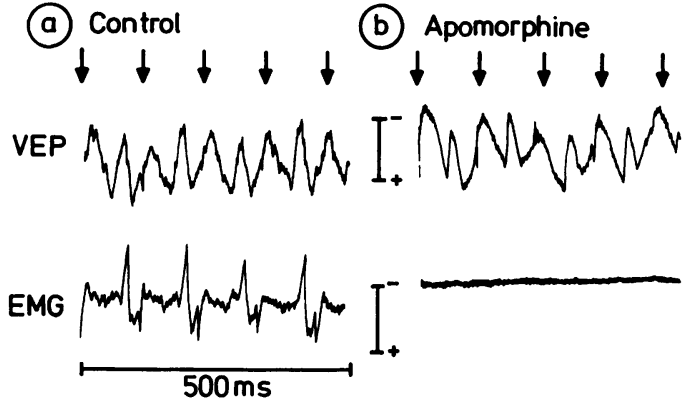

(c)

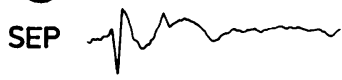

(d)
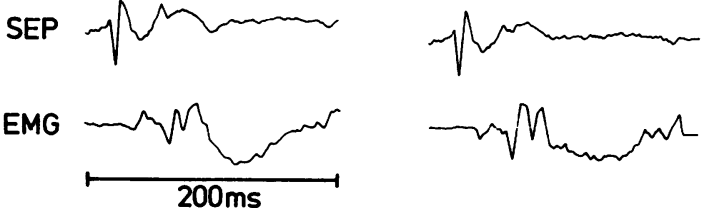

Fig 3 Case 1. Visual evoked potentials (VEP) to flash stimulation (arrows) at a frequency of $10 \mathrm{~Hz}$, somatosensory evoked potentials (SEP) to digital nerve stimulation of the right forefinger and electromyographic (EMG) recording of the reflex jerks from right finger flexor muscles. Each trace is the average of 32 responses. $(A)$ and (C) control records. (B) and (D) after $1 \mathrm{mg}$ of subcutaneous apomorphine. Reflex myoclonus to flash stimulation was abolished by apomorphine, but electrically elicited myoclonus was not decreased. Notice that the amplitude of the primary complex of the VEPs remains constant. The baseline of the cortical record changed slightly after apomorphine; this variation was probably due to the absence of muscle artefact once the reflex myoclonus had disappeared. Vertical calibration bars are $50 \mu V$ for VEPs, $25 \mu V$ for SEP and $200 \mu V$ for EMG potentials.

axonal swelling ("'torpedoes") from Purkinje cells were observed. The dentate nucleus and its ribbon as well as the other deep cerebellar nuclei were preserved. At the level of the medulla (fig 2-F) relevant abnormalities comprised severe neuronal loss and fibrillary gliosis of the inferior olives and arciform nucleus, the dorsal motor nucleus of the vagus, and the hypoglosal nucleus. Moderate demyelination of the spino-cerebellar tracts was present. The spinal cord sections showed marked neuronal loss and gliosis of the anterior and lateral horns with mild gliosis of Clarke's columns.

\section{Pharmacological and physiological studies}

1 Control Flash stimulation at a frequency of 10 to $20 \mathrm{~Hz}$ elicited rhythmical myoclonus. At these frequencies each flash produced a large VEP (50 $\mu \mathrm{V})$ followed by a generalised jerk which caused an artifact in the scalp (occipital) electrode (fig 3A).
The latency of the first positive peak of the VEP was $32 \mathrm{~ms}$ and the time interval between this peak and the onset of the reflex EMG discharge recorded from biceps brachii was $28 \mathrm{~ms}$. SEPs were also enlarged $(35 \mu \mathrm{V}, \mathrm{P} 1-\mathrm{N} 2)$ and were followed by a reflex EMG discharge with a latency of $40 \mathrm{~ms}$ after the stimulus recorded from biceps brachii (fig $3 \mathrm{C}$ ).

2 Apomorphine A single subcutaneous dose of $1 \mathrm{mg}$ of apomorphine was given prior to intravenous administration of $40 \mathrm{mg}$ of domperidone. Clinical and electrophysiological evaluation of myoclonus was repeated 30 minutes after apomorphine. The myoclonic jerking previously provoked by flash stimulation at a frequency of 10 to $20 \mathrm{~Hz}$ was abolished following apomorphine, but the amplitude and morphology of the primary complex of the VEP remained constant (fig 3B). The slight change in morphology of the cortical record was probably due to the absence of muscle artefact. On this occasion the flash could be kept on indefinitely without eliciting any reflex myoclonus. In contrast, the amplitude of the EMG myoclonic discharge produced by electrical stimulation of the fingers was not modified by apomorphine (fig 3D).

3 Lisuride A single intravenous dose of $0.1 \mathrm{mg}$ of lisuride was given on a different day. Twenty minutes later, a striking reduction in visual reflex $\underset{\mathbb{D}}{\forall}$ myoclonus was observed. However, rhythmical $\stackrel{\rho}{\mathrm{D}}$ 윽

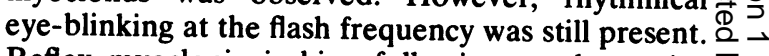
Reflex myoclonic jerking following touch or pin- 0 prick was decreased and the size of the EMG reflex discharge elicited by electrical stimulation was diminished to half of the control value.

Case 2. PRL (Clínica Universitaria, No 73247).

This 67-year-old lady was diagnosed as having Parkinson's disease in 1971. She responded initially to levodopa therapy and levodopa plus benserazide subsequently. In 1975, akinesia and rigidity had become prominent and a "wearing off phenomenon" was present. Bromocriptine and amantadine were added but did not produce any greater improvement. When examined in 1983 , she was unable to speak, required assistance to walk a few metres and was severely rigid. There was also marked dysphagia. Tendon jerks were exaggerated and a right Babinski sign was present. Pursuit ocular movements were abnormal (cogwheeling) and ocular dysmetria was observed upon attempted saccadic movements of the eyes. Dysmetria of the upper limbs in the finger-to-nose test was noticed, evaluation of coordination in the lower limbs was made difficult by severe akinesia. Light touch or pin-prick to the fingers and toes, or tapping the wrist or toes with a tendon hammer, provoked repetitive muscle jerks localised to the stimulated limb. In the arm 

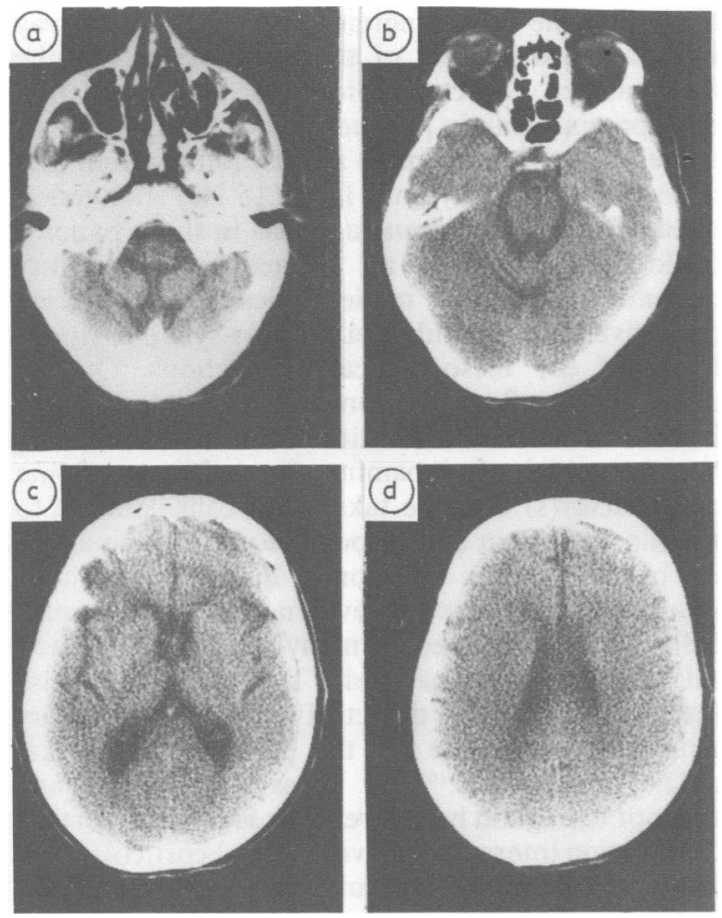

Fig 4 CT brain scan of patient No 2. Marked brainstem and cerebellar atrophy, the latter mainly due to atrophy of the vermis, is observed ( $A$ and $B$ ). There is also slight dilatation of the third ventricle and anterior horns $(C)$, without cortical atrophy (D).

these were characterised by flexion of fingers, wrist and elbow; in the legs by flexion of the toes, and flexion/extension of the great toe. Treatment with levodopa-carbidopa was discontinued for a few days with only slight deterioration in her motor capacity. At this time, the flashing stimulus employed for routine EEG recording elicited repetitive generalised myoclonic jerking unaccompanied by loss of conciousness. The CT scan showed mark atrophy of the brainstem and cerebellar vermis with only moderate cortical atrophy (fig 4). A clinical diagnosis of sporadic olivo-ponto-cerebellar atrophy was made. She was treated with lisuride $(3 \mathrm{mg} /$ day p.o.) and levodopa-carbidopa 275 ( 4 tablets/day p.o.) without further improvement. A few months later she died of aspiration pneumonia at home.

\section{Pharmacological and physiological studies}

1 Off levodopa therapy. Flash stimulation at a frequency of $3 \mathrm{~Hz}$ (or greater) induced generalised and rhythmical myoclonus (fig 5A). Each flash produced a large VEP $(50 \mu \mathrm{V})$ which was followed 30 ms later by a generalised jerk. SEPs were also increased in amplitude $25 \mu \mathrm{V}(\mathrm{P} 1-\mathrm{N} 2)$ and were associated with reflex EMG discharges occurring with a latency of $40 \mathrm{~ms}$ in finger flexors.

2 On levodopa therapy The electrophysiological studies were repeated three days after Sinemet 275 (levodopa $250 \mathrm{mg}$, carbidopa $25 \mathrm{mg}$ ) (4 tablets/day) had been restarted. At that time, flashing at any frequency $(3$ to $50 \mathrm{~Hz})$ during a prolonged period of time (up to 30 seconds) failed to elicit any reflex myoclonus. However VEPs were still enlarged (fig 5B). Reflex myoclonic jerking triggered by electrical stimulation was unchanged.

3 Lisuride $0.15 \mathrm{mg}$ of lisuride was given IV when the patient was taking her usual dose of Sinemet. Accordingly only the effect of lisuride on somaesthetically induced myoclonus could be studied. A marked reduction (90\%) in the amplitude of the EMG reflex discharges recorded in finger flexors following digital nerve stimulation was found, which corresponded with diminution in reflex myoclonus in the limbs observed clinically.

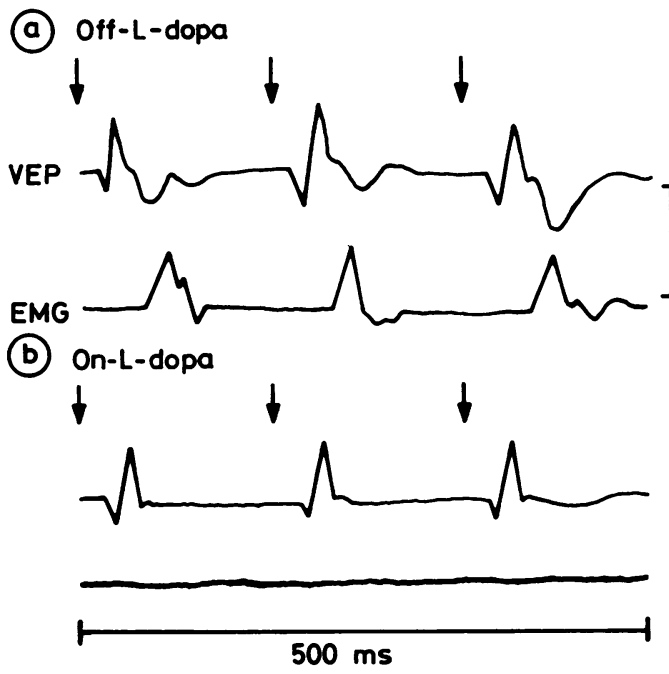

Fig 5 Case 2. (A) Visual evoked potentials (VEPs) and reflex EMG discharges recorded from right biceps brachii to flash stimulation (arrows) at a frequency of $6 \mathrm{~Hz}$, while the patient was not receiving any dopamine agonist. (B) Following oral treatment with levodopa-carbidopa (1000/100 mg/day). The reflex muscle responses were abolished after levodopa without change in the amplitude of the VEPs. The late positive wave observed in the control $V E P$ represented a muscle artefact due to the reflex myoclonus; accordingly it is not present after treatment. Vertical bar is $50 \mu V$ for VEP and $500 \mu V$ for EMG trace. 


\section{Discussion}

Dopamine agonists abolished cortical reflex myoclonus to flash stimulation in two patients with olivo-ponto-cerebellar atrophy. These results extend the findings of Quesney et al ${ }^{1314}$ of apomorphine-induced suppression of photosensitive epileptic discharges in humans, and support experimental work indicating a strong inhibitory action of dopamine agonists upon the photomyoclonic response in the baboon Papio papio. ${ }^{12}$ The site and mechanism of action of dopamine agonist inhibition of the abnormal cortical discharges producing myoclonus is not clear. Dopamine agonists did not reduce the amplitude of the VEPs preceding the myoclonic jerks in either of the two patients, indicating that the antimyoclonic effect of these drugs was not due to inhibition of the afferent visual impulses at the retina or lateral geniculate bodies. The abnormal occipital discharges evoked by flash stimulation could have spread via occipito-reticular pathways or utilising the dense visuo-motor (occipitalpremotor cortex) connections. ${ }^{20}$ The former possibility seems unlikely in view of (a) the short latency $(30 \mathrm{~ms})$ between the primary complex of the VEPs and the myoclonic jerking in the arms, and (b), the small size and significance of visuo-reticular pathways in humans. ${ }^{21}$ On the other hand a corticocortical link is favoured by experimental findings in the photosensitive baboon ${ }^{22} 23$ and in a recent patient with visual reflex myoclonus studied by Shibasaki, ${ }^{24}$ in whom evidence for occipito-frontal transmission of the abnormal discharges generated by flash stimulation was obtained. Interestingly, the occipito-frontal conduction time $(10 \mathrm{~ms})$ recorded in Shibasaki's patient coincided almost exactly with our indirect calculations $(10-12 \mathrm{~ms})$ in the two patients reported here. In addition, in these two patients, cortical reflex myoclonus to somaesthetic stimuli was not modified by apomorphine or levodopa. It is therefore unlikely that dopamine agonists suppressed visual reflex myoclonus by way of a nonspecific inhibitory action or by decreasing the excitability of cortico-spinal motoneurons. Accordingly we propose that the antimyoclonic effect of the dopamine agonist was probably due to a selective inhibition of visual cortex output neurons (area 19) projecting onto the premotor cortex or inhibiting the "premotor" cortex neurons by which visual cortex neurons communicate with motoneurons in area $4 .^{2022}$

The main source of cortical dopamine arises from the basal ganglia via the meso-cortical dopaminergic pathway. ${ }^{25}$ In patient 1 , and probably in patient 2 , there was severe damage of the entire substantia nigra and surrounding structures. It is therefore likely that the dopaminergic meso-cortical pathway was damaged as part of the multisystem atrophy they suffered. Direct microiontophoretic application of dopamine mainly produces neuronal inhibition ${ }^{26}$ and endogenous dopamine activity in the visual cortex is reduced by rhythmic flash $(15 \mathrm{~Hz})$ stimulation in cats. ${ }^{27}$ Thus, dopamine agonists in these patients could have restored an intracortical inhibitory dopaminergic defect, made clinically overt during repetitive visual stimulation, by post-synaptic stimulation of the meso-cortical connections. On the other hand, reflex myoclonus of any type is not a feature of untreated Parkinson's disease, the best human model of dopaminergic deficiency. Some other factor(s) must be taken into consideration to explain the origin and response to dopamine agonists of visual reflex myoclonus. In the two patients described here there was evidence of marked cerebellar damage. Indeed, in many patients with cortical myoclonus, clinical and CT scan features raise the possibility that a defect of cerebellar inhibitory output is responsible for the pathological cortical reflex mechanisms. ${ }^{16}$ At present however, any attempt to explain how cerebellar and meso-cortical dysfunction interact to provoke visual corticol myoclonus would be mere speculation.

The findings reported here confirm previous results indicating that pure dopamine agonists are $\stackrel{\leftrightarrow}{\stackrel{9}{0}}$ 을

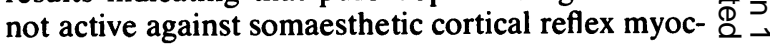
lonus. ${ }^{17}$ Unfortunately, it was not possible to test the $\overline{0}$ effect of 5-HTP plus carbidopa upon the visual $\varnothing$ reflex myoclonus present in our two patients. Experimental evidence suggests that serotonin agonists are also capable of suppressing photomyoclonic and photoconvulsive responses. ${ }^{28}$ Whether or not this is the case in humans requires further investigation.

Cortical reflex myoclonus is difficult to treat and may be associated with generalised seizures. ${ }^{16}$ Appropiate control of visually triggered myoclonus often improves the standard of living of patients with myoclonic epilepsy. Dopamine agonists might be considered as an additional therapeutic tool in patients with photic epilepsy.

The authors are grateful to Mrs MA García and Mrs M Obeso for technical help. Mrs ML Sola patiently typed the manuscript.

\section{References \\ ${ }^{1}$ Lhermitte F, Petrafaldi M, Marteau R, Gazengel J, Ser- dam $M$. Analyse pharmacologique d'un cas de myo- clonies d'intention et d'action post-anoxiques. $\mathbf{R e v}$ Neurol (Paris) 1971;124:21-31. \\ ${ }^{2}$ Lhermitte R, Marteau R, Degos CF. Analyse phar- macologique d'un nouveau cas de myoclonies d'inten-}


tion et d'action post-anoxiques. Rev Neurol (Paris) 1972;126: 107-14.

${ }^{3}$ Chadwick D, Hallett M, Harris R, Jenner P, Reynolds EH, Marsden CD. Clinical, biochemical, and physiological features distinguishing myoclonus responsive to 5-hydroxytryptophan, tryptophan with a monoamine oxidase inhibitor and Clonazepam. Brain 1977; 100:455-87.

${ }^{4}$ Growdon JH, Young RR, Shahani T. L- 5 hydroxytryptophan in treatment of several different syndromes in which myoclonus is prominent. Neurology (Minneap) 1976;26: 1135-40.

${ }^{5}$ Van Woert MH, Sethy VH. Therapy of intention myoclonus with L-5 hydroxytryptophan and a peripheral decarboxylase inhibitor, MK 486. Neurology (Minneap) 1975;25: 135-40.

- Van Woert MH, Rosenbaum D, Howilson J, Bowers MB. Long-term therapy of myoclonus and other neurologic disorder with L-5-hydroxytryptophan and Carbidopa. N Engl J Med 1977;296:70-75.

${ }^{7}$ Chadwick D, Harris R, Jenner P, Reynolds EH, Marsden CD. Manipulation of brain serotonin in the treatment of myoclonus. Lancet 1975;2:434-5.

${ }^{8}$ Van Woert MH, Rosenbaum D. L-5-hydroxytryptophan therapy in myoclonus. In: Fahn S, Davis JN, Rowland PL, eds. Advances in Neurology, Vol. 26. New York: Raven Press. 1979: 109-22.

' Glatt S, Klawans HL, Weiner WJ, Prelevic S. Myoclonic disorders responsive to serotoninergic blockade. Neurology (Minneap) 1979;29:606-7.

${ }^{10}$ Thal LJ, Sharpless NS, Wolfson L, Katzman R. Treatment of myoclonus with L-5-Hydroxytryptophan and Carbidopa: Clinical, electrophysiological, and biochemical observations. Ann Neurol 1980; 7:570-6.

${ }^{11}$ Meldrum BS. Mode of action of anticonvulsant drugs: biochemical effects. The treatment of epilepsy. In: Tyrer JH, ed. Current Status of Modern Therapy. Vol. 5. Lancaster: MTP Press, 1980:29-59.

12 Meldrum BS. Photosensitive epilepsy in Papio papio as a model for drug studies. In: Cobb WA, Van Duijn $\mathrm{H}$, eds. Contemporary Clinical Neurophysiology (EEG Suppl. 34), Amsterdam: Elsevier, 1978:317-22.

${ }^{13}$ Quesney LF, Andermann F, Lal L, Nauseida PA. Transient abolition of generalized photosensitive epileptic discharge in humans by apomorphine, a dopamine receptor agonist. Neurology (Minneap) 1980; 30:1169-74.
14. Dawson GD. Investigations on a patient subject to myoclonic seizures after sensory stimulation. J Neurol Neurosurg Psychiatry 1977;10:141-62.

${ }^{15}$ Hallett M, Chadwick D, Marsden CD. Cortical reflex myoclonus. Neurology (Minneap) 1979;29:1107-25.

${ }^{16}$ Obeso JA, Rothwell JC, Marsden CD. The spectrum of cortical myoclonus. Brain 1985; 108: 193-224.

${ }^{17}$ Obeso JA, Rothwell JC, Quinn NP, Lang AC, Thompson $C$, Marsden $C D$. Cortical reflex myoclonus responds to intravenous Lisuride. Clin Neuropharmacology 1983;6:231-40.

${ }^{18}$ Rothwell JC, Obeso JA, Marsden CD. On the significance of giant somatosensory evoked potentials in cortical myoclonus. J Neurol Neurosurg Psychiatry 1984;47:33-42.

${ }^{14}$ Quesney LF, Andermann F, Gloor P. Role of a dopaminergic mechanism in generalized photosensitive epilepsy. Neurology (NY) 1981;31:1542-4.

${ }^{20}$ Haaxma R, Kuypers HGJM. Intrahemispheric cortical connexional and visual guidance of hand and finger movements in the rhesus monkey. Brain 1975;98:239-60.

${ }^{21}$ Brodal A. Neurological Anatomy. New York, Oxford University Press, 1981.

${ }^{22}$ Menini CH. Role du cortex frontal dans l'épilepsie photosensible du singe Papio papio. J Physiol (Paris) 1976; 72:5-44.

${ }^{23}$ Catier J, Charmasson G, et Christolomme A. Study of ipsilateral cortico-cortical connections from the occipital lobe in the photosensitive baboon. J Physiol (Paris) 1973;66:93-100.

${ }^{24}$ Shibasaki H. In: Fahn S, Marsden CD, Van Woert M, eds. Myoclonus. New York: Raven Press, in press.

${ }^{25}$ Moore RY, Bloom FE. Central catecholamine neuron systems: anatomy and physiology of the dopamine systems. Ann Rev Neurosci 1978;1:129-169.

${ }^{26}$ Reader TA, Ferron A, Descarries L, Jasper HH. Modulatory role for biogenic animes in the cerebral cortex. Microiontophoretic studies. Brain Res 1979; 160:217-29.

${ }^{27}$ Reader TA, De Champlain J, Jasper H. Catecholamines released from cerebral cortex in the cat: decrease during sensory stimulation. Brain Res 1976;111:95-108.

${ }^{28}$ Wada JA, Balzamo E, Meldrum BS, Naquet R. Drugs modifying brain serotonin content and photosensitivity in the Senegalese baboon (Papio papio). Electroencephalogr Clin Neurophysiol 1972;33:520-27. 